Nota de investigación

\title{
Efecto del ácido salicílico en la germinación y crecimiento radicular del tomate
}

\author{
Gabriela Dzib-Ek ${ }^{1}$ \\ Eduardo Villanueva-Couoh ${ }^{1}$ \\ René Garruña-Hernández ${ }^{1}$ \\ Silvia Vergara Yoisura ${ }^{2}$ \\ Alfonso Larqué-Saavedra ${ }^{2 \S}$
}

${ }^{1}$ División de Estudios de Posgrado e Investigación-Instituto Tecnológico de Conkal. Avenida Tecnológico s/n, Conkal, Yucatán, México. CP. 97345. Tel. 9999124130. (gabriela_capuleto@hotmail.com; eduardo.villanueva@itconkal.edu.mx; renegh10@hotmail.com). ${ }^{2}$ Recursos Naturales-Centro de Investigación Científica de Yucatán. Calle 43 núm. 130 x 32 y 34. Chuburná de Hidalgo, Mérida, Yucatán, México. CP.97205. Tel.9999428330. (silvana@ cicy.mx).

${ }^{\S}$ Autor para correspondencia: larque @ cicy.mx.

\section{Resumen}

El tomate (Solanum lycopersicum L.), es una hortaliza perteneciente a la familia de las solanáceas. Este cultivo es importante en varios países, principalmente por su alto valor económico reflejado en su gran demanda, con mercados para consumo fresco o industrializado. Debido a su importancia comercial, se realizan investigaciones de sus cultivos, para obtener plántulas de buena calidad. El ácido salicílico ha sido propuesto como un regulador de crecimiento vegetal, debido a los efectos inducidos en algunos procesos fisiológicos de las plantas. El objetivo de este estudio fue evaluar el efecto de diferentes concentraciones de ácido salicílico sobre la germinación y calidad de plántulas de tomate. Las pruebas de imbibición de las semillas y la preparación del ácido salicílico se realizaron en el laboratorio de fisiología y biotecnología vegetal del Intituto Tecnológico de Conkal, Yucatán, durante 2016-2017. Se utilizaron semillas de tomate variedad Río Grande con hábito de crecimiento determinado. Las semillas se sometieron a un proceso de imbibición durante $24 \mathrm{~h}$ en condiciones de laboratorio controladas. Los tratamientos evaluados fueron $0,1,0.01 \mathrm{y}$ $0.0001 \mu \mathrm{M}$ de ácido salicílico (AS) y como control uno sin imbibición. Con los resultados se realizó un análisis de varianza, así como la prueba de comparación de medias por el método de Tukey $(p \leq$ 0.05), mediante el paquete estadístico SAS ver 9.3. Los resultados demostraron que el tiempo de imbibición de semillas en concentraciones de ácido salicílico no inhibe la germinación y estimula la diferenciación de raíces secundarias en concentraciones de 1 y $0.01 \mu \mathrm{M}$ AS.

Palabras clave: Solanum lycopersicum L., ácido salicílico, germinación.

Recibido: marzo de 2021

Aceptado: abril de 2021 
El tomate (Solanum lycopersicum L.) es uno de los cultivos más importantes a nivel muncial debido a su consumo tanto en fresco como en productos procesados. Para un buen rendimiento de esta hortaliza es importante la obtención de plántulas de buena calidad, que tengan un mejor crecimiento radicular, lo que favorece una mejor producción de tomate (Calero et al., 2019).

La germinación es una de las etapas más importantes en el proceso de crecimiento y desarrollo de la planta, la cual comienza con la toma de agua por la semilla en un proceso llamado imbibición. El uso de reguladores de crecimiento vegetal (RCV), como el ácido salicílico (AS) que se encuentra en todos los tejidos vegetales (Martín et al., 2015) son consideradas una alternativa para incrementar la producción de alimentos de importancia hortícola y cereales ya sea bajo condiciones de invernadero o a cielo abierto (Martín et al., 2013).

En Capsicum chinense el AS incrementa el tamaño de las raíces, favoreciendo la absorción y acumulación de macro y micronutrientes (Tuchuch-Haas et al., 2019) así como otros estudios proponen al ácido salicílico como alternativa viable para incrementar la producción y calidad nutracéutica en tomate (Vázquez-Diaz et al., 2016).

Martín et al. (2013), reportan los efectos beneficos del ácido salicílico en la producción de variedades de tomate, pepino, chile habanero, pimiento y otras especies. La evaluación foliar de AS en plántulas de tomate y pimiento favorece determinadas variables de crecimiento y contenido mineral (Valdez-Sepúlveda et al., 2015). Plantas de tomate provenientes de semillas tratadas en concentraciones de ácido salicílico, mostraron un efecto positivo, en el área foliar, longitud de raíz, tamaño de frutos, así como el rendimiento por planta (Rodríguez-Larramendi et al., 2008).

En otras especies como Phaseolus vulgaris L, el tiempo de imbibición de las semillas determina el efecto del AS (Rodríguez-Larramendi et al., 2017). Sin embargo, hay reportes (BenavidesMendoza, et al., 2004; Rodríguez-Larramendi et al., 2017) de que este regulador vegetal presenta un efecto inhibitorio sobre la germinación en algunas hortalizas a determinadas concentraciones y periodos de imbibición. El objetivo de esta investigación fue evaluar en tomate el efecto de la imbibición de ácido salicílico en la germinacion de las semillas y medir si este reguador del crecimiento afecta el crecimiento de las raices de las plántulas.

El estudio se realizó en el Instituto Tecnológico de Conkal, Yucatán. Las pruebas de imbibición de las semillas y la preparación del ácido salicílico se realizaron en el laboratorio de fisiología y biotecnología vegetal durante 2016-2017. Se utilizaron semillas de tomate variedad comercial Río Grande con hábito de crecimiento determinado. Para evaluar la germinación, lotes de semillas se colocaron en bolsas de tela manta de cielo, se imbibieron en cada solución correspondiente a cada tratamiento durante $24 \mathrm{~h}$ en un cuarto de crecimiento bajo condiciones controladas, (temperatura $21 \pm 1^{\circ} \mathrm{C}$, humedad relativa $30 \%$ ).

Los tratamientos evaluados fueron $0 \mu \mathrm{M}, 1 \mu \mathrm{M}, 0.01 \mu \mathrm{M}$ y $0.0001 \mu \mathrm{M}$ de AS y un testigo sin imbibición. Para preparar las tres concentraciones de AS se siguió la metodología descrita por Gutiérrez-Coronado et al. (1998). Los bioensayos para evaluar la germinación y el crecimiento de las radiculas se realizaron siguiendo la tecnica descrita por Larqué-Saavedra et al. (1975). El ensayo consistió en sembrar las semillas de tomate sobre mallas de tela en frascos de vidrio, con $250 \mathrm{ml}$ de agua destilada o la solución de AS correspondiente a cada tratamiento. 
Los frascos se mantuvieron en un cuarto de germinación bajo condiciones controladas. Se estableció un diseño experimental completamente al azar con cinco tratamientos $(0,1,0.01,0.0001$ $\mu \mathrm{M}$ de AS y control sin imbibición). Se utilizaron 20 semillas por repetición, cinco repeticiones por tratamiento. Siete días despues de iniciado el experimento se evaluo el porcentaje de germinación y la longitud radicular (a través del programa ImageJ), para esta variable se midieron 10 plántulas por cada tratamiento.

Con los resultados se realizó un análisis de varianza, así como la prueba de comparación de medias por el método de Tukey $(p \leq 0.05)$, mediante el paquete estadístico SAS ver 9.3. No se pudo apreciar efecto alguno de inhibicion por AS en la germinacion de las semillas de tomate en los dos ensayos. En el bioensayo uno el porcentaje de germinación oscilo de $87 \%$ a $94 \%$. El porcentaje de germinación en el bioensayo dos presento de $89 \%$ a $97 \%$, en comparación con el tratamiento de semillas sin imbibición o enbebidas con agua destilada). El analisis reporta que no hay diferencias estadísticamente diferentes entre tratamientos (Tukey, $p \leq 0.05$ ).

Estos resultados presentaron respuestas similares a lo reportado por García-Osuna et al. (2015) que en tomate verde (Physalis ixocarpa) el ácido salicílico no inhibe la germinación y estimula este proceso fisiológico desde el primer día de la siembra en concentraciones $10^{-2} \mathrm{M}, 10^{-4} \mathrm{M}$ y $10^{-6} \mathrm{M}$. Es importante señalar que concentraciones $10^{-2}$ y $10^{-3} \mathrm{M}$ de AS reportadas por Benavides et al. (2004), inhiben la germinación de semillas de chile Tampiqueño (Capsicum annuum) variedad 74. Utilizando concentraciones bajas de $10^{-5} \mathrm{M}$ se reportan datos de germinación con un comportamiento similar al testigo.

Este comportamiento sugiere que el efecto de las concentraciones de AS depende del tiempo de exposición a este regulador de crecimiento vegetal, lo cual probablemente incrementa los niveles endógenos de ácido abscísico y de este modo inhibe la germinación (Rodríguez-Larramendi et al., 2017). Los resultados obtenidos confirman que los pretratamientos germinativos son una herramienta que incrementa porcentaje de germinación.

La longitud radicular de las plántulas cultivadas en agua destilada que provenian de semillas embebidas durante $24 \mathrm{~h}$ en una de las diferentes concentraciones de AS de tomate se evaluó a los de siete días. El análisis estadístico del bioensayo indica diferencias significativas entre los tratamientos. El tratamiento de semillas embebidas en agua destilada y semillas sembradas directamente tienen los menores valores y las concentraciones $0.01 \mathrm{y} 0.0001 \mu \mathrm{M}$ AS arrojaron el mayor efecto estimulante (Figura 1).

La longitud radicular de las plántulas de semillas sin pretratamiento de AS que fueron cultivadas en soluciones de diferentes concentraciones de AS o agua destilada durante siete dias, se muestra en la (Figura 2). El análisis estadístico del bioensayo indica diferencias significativas entre los tratamientos. Los resultados del ensayo muestran que cuando las raíces se cultivan en la solucion con $0.01 \mu \mathrm{M}$ AS, presentan mayor longitud radicular en comparación con los demás tratamientos.

De acuerdo a lo reportado por Larqué-Saavedra et al. (2010), las plántulas tratadas con AS en concentraciones 0.01 y $1 \mu \mathrm{M}$ favorecen del desarrollo de la raíz de Lycopersicon esculentum Mill. Las aplicaciones de bajas concentraciones de ácido salicílico a los brotes de plántulas de plantas 
hortícolas como del género Capsicum refleja su efecto positivo en el crecimiento, desarrollo de la planta, así como crecimiento de las raíces que correlaciona una mayor absorción de macro y micronutrimentos que se asignan en los tejidos vegetales (Tucuch-Haas et al., 2017).

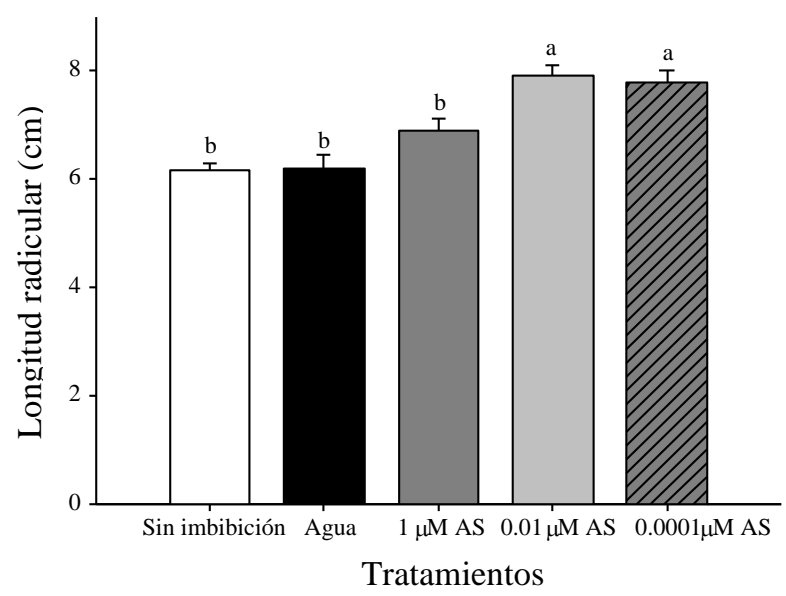

Figura 1. Longitud radicular de plántulas de tomate cultivadas durante siete dias en agua destilada. Dichas plántulas provenían de semillas embebidas durante $24 \mathrm{~h}$ en una de las diferentes concentraciones de ácido salicílico señalados en la gráfica de semillas embebidas en agua destilada o semillas sembradas directamente. Literales diferentes indican diferencia estadística significativa (Tukey, $p \leq 0.05)$. Los datos son medias \pm error estándar $(n=10)$.

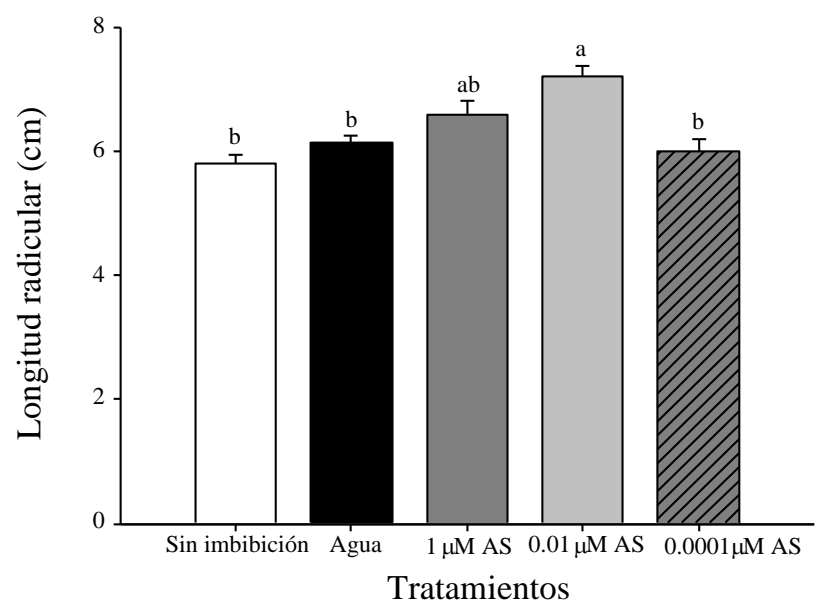

Figura 2. Longitud radicular de plántulas de tomate variedad Río Grande cultivadas durante siete días en una de las diferentes concentraciones de ácido salicílico señalados en la gráfica o en agua destilada. Literales diferentes indican diferencia estadística significativa (Tukey, $p \leq 0.05$ ). Los datos son medias \pm error estándar $(n=10)$.

Cabe resaltar, que se observó que las raíces cultivadas en las soluciones de diferentes concentraciones de AS presentaron formación de raíces secundarias, efecto que no se manifestó en las raíces cultivadas solo en agua o las de las semillas de siembra directa (Figura 3). 


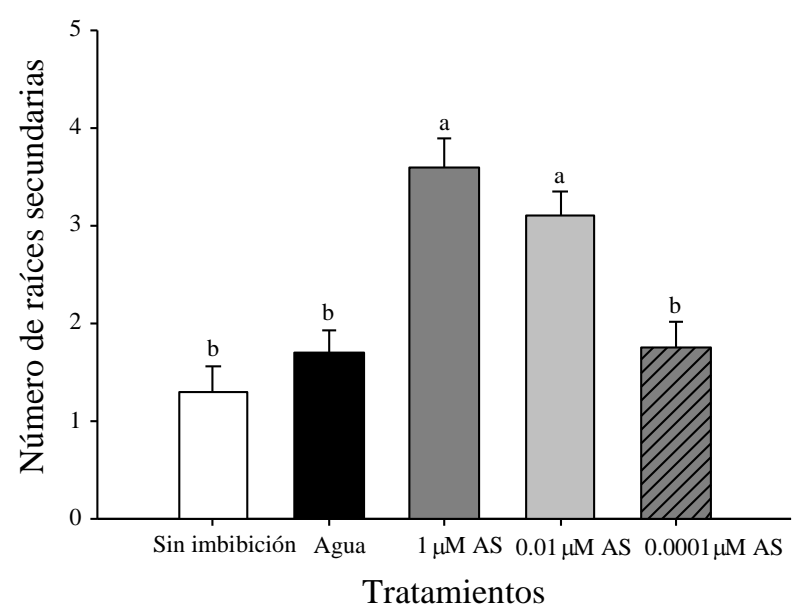

Figura 3. Número de raíces secundarias de plántulas de tomate variedad Río Grande cultivadas durante siete dias en una de las diferentes concentraciones de ácido salicílico señalados en la gráfica o en agua destilada. Literales diferentes indican diferencia estadística significativa (Tukey, $p \leq 0.05)$. Los datos son medias \pm error estándar $(\mathrm{n}=10)$.

La aplicación de AS a las plantas de Capsicum annuum L. cv Chichimeca en contacto con AS en concentraciones de 0.1 y $0.2 \mathrm{mM}$ manifiesta un aumento en la producción de biomasa foliar, en raíz y de frutos (Sánchez-Chávez et al., 2011).

Los resultados del presente estudio, coinciden con lo publicado por autores como Vázquez-Diaz et al. (2016), quienes reportaron el efecto significativo en el rendimiento de tomate con dosis 0.025 y $0.1 \mathrm{mM}$ de AS al ser diluido en una solución nutritiva.

Guzmán-Antonio et al. (2012) mencionan que el AS en concentración de $10^{-8} \mathrm{M}$ en sinergia con fertilización de $\mathrm{N}, \mathrm{P}$ y K $\left(190 \mathrm{mg} \mathrm{L}^{-1}\right.$ de $\left.\mathrm{N}-\mathrm{P}_{2} \mathrm{O}_{5}-\mathrm{K}_{2} \mathrm{O}\right)$ incrementaron la altura, número de hojas y longitud radical específica de las plántulas en chile habanero. El efecto de la aplicación exógena del AS en el crecimiento de las plantas, depende de la especie, la etapa de desarrollo y la concentración aplicada (Rivas y Plascencia, 2011). Con base a los resultados el AS representa una alternativa en germinación y producción de tomate.

\section{Conclusiones}

La imbibición de las semillas de tomate en ácido salicílico (1, 0.01 y $0.0001 \mu \mathrm{M})$ durante 24 h no inhibe su germinación. El ácido salicílico en concentraciones 1 y $0.01 \mu \mathrm{M}$ favorece significativamente la longitud radicular de las plántulas y la formación de raíces secundarias en comparación con los controles.

\section{Literatura citada}

Benavides-Mendoza, A.; Salazar-Torres, A. M.; Ramírez-Godina, F.; Robledo-Torres, V.; Ramírez-Rodríguez, H. y Maiti, R. 2004. Tratamiento de semilla de chile con ácidos salicílico y sulfosalicílico y respuesta de las plántulas al frío. Terra Latinoam. 22(1):41-47. 
Calero, H. A.; Quintero, R. E.; Pérez, D. Y.; Olivera,V. D.; Peña, C. K.; Castro, L. I. y Jiménez, H. J. 2019. Evaluación de microorganismos eficientes en la producción de plántulas de tomate (Solanum lycopersicum L.). Rev. Mex. Cienc. Agric 36(1):67-78.

García-Osuna, H. T.; Escobedo-Bocardo, L.; Robledo-Torres, V.; Benavides-Mendoza, A. y Ramírez-Godina, F. 2015. Germinación y micropropagación de tomate de cáscara (Physalis ixocarpa) tetraploide. Rev. Mex. Cienc. Agric. 6(2):2301-2311.

Gutiérrez-Coronado, M.; Trejo, C. y Larqué-Saavedra, A. 1998. Effect of salicylic acid on the growth of roots and shoots in soybean. Plant Physiol Biochem. 36(8):563-565.

Guzmán-Antonio, A.; Borges-Gómez, L.; Pinzón-López, L.; Ruiz-Sánchez, E. y Zuñiga-Aguilar, J. 2012. Efecto del ácido salicílico y la nutrición mineral sobre la calidad de plántulas de chile habanero. Agrom. Mesoam. 23(2):247-257.

Larqué-Saavedra, A.; Wilkins, H. and Wain, R. L. 1975. Promotion of cress root elongation in white light by 3, 5 diiodo-4-hydroxybenzoic acid. Planta. 126(3):269-272.

Larqué-Saavedra, A.; Martín, R.; Nexticapan-Garcéz, A.; Vergara-Yoisura, S. y Gutiérrez Rendón, M. 2010. Efecto del ácido salicílico en el crecimiento de plántulas. (Lycopersicon esculentum Mill.). Rev. Chapingo Ser. Hortic. 16(3):183-187.

Martín, R.; Nexticapan-Garcéz, A. and Larqué Saavedra, A. 2013. Potential benefits of salicylic acid in food production. In: salicylic acid. Hayat, S.; Ahmad, A. and Alyemeni, M. N. (Ed.). Springer publishers. Dortdrech, the Netherlands. 299-313 p.

Martín, R.; Larqué-Saavedra, A.; Vergara-Yoisura, S.; Uicab-Quijano, V.; Villanueva-Couoh, E. 2015. Ácido salicílico estimula la floración en plantas micropropagadas de gloxínia. Rev. Fitotec. Mex. 38(2):115-118.

Rivas-San, V. M. and Plasencia, J. 2011. Salicylic acid beyond its role in plant growth and development. J. Exp. Bot. 1(10):1-18.

Rodríguez-Larramendi, L.; Matos, Y.; Santos, P. e Infante, S. 2008. Crecimiento, floración y fructificación en plantas de tomate (Lycompersicom esculentum L. var. Vyta) provenientes de semillas tratadas con ácido salicílico. Centro Agrícola. 35:29-34.

Rodríguez-Larramendi, L.; González-Ramírez, M.; Gómez-Rincón, A.; Guevara-Hernández, F.; Salas-Marina, M. y Gordillo-Curiel, A. 2017. Efectos del ácido salicílico en la germinación y crecimiento inicial de plántulas de frijol (Phaseolus vulgaris L.). Rev. Facult. Agron. 34(3):253-269.

Sánchez-Chávez, E.; Barrera-Tovar, R.; Muñoz-Márquez, E.; Ojeda-Barrios, D. L. y AnchondoNájera, A. 2011. Efecto del ácido salicílico sobre biomasa, actividad fotosintética, contenido nutricional del chile jalapeño. Rev. Chapingo Ser. Hortic. 17(1):63-66.

Tucuch-Haas, C. J.; Pérez-Balam, J. V.; Díaz-Magaña, K. B.; Castillo-Chuc, J. M.; Dzib-Ek, M. G.; Alcántar-González, G.; Vergara-Yoisura, S. and Larqué-Saavedra, A. 2017. Role of salicylic acid in the control of general plant growth, development, and productivity. In: salicylic acid: a multifaceted hormone. Nazar, R.; Iqbal, N. and Khan, N. (Ed.). Springer Nature, Singapore. 1-15 p.

Tucuch-Haas, C. J.; Pérez-Balam, J. V.; Dzib-Ek, M. G.; Alcántar-González, G. y LarquéSaavedra, A. 2019. El ácido salicílico aumenta la acumulación de macro y micronutrientes en chile habanero. Rev. Mex. Cienc. Agric. 10(4):839-847.

Valdez-Sepúlveda, L.; González-Morales, S.; Valdez-Aguilar, L. A.; Ramírez-Godina, F y Benavides-Mendoza, A. 2015. Efecto de la aplicación exógena de ácido benzoico y salicílico en el crecimiento de plántulas de tomate, tomatillo y pimiento. Rev. Mex. Cienc. Agric. 6(2):2331-2343.

Vázquez Diaz, D. A.; Salas Pérez, L.; Preciado Rangel, P.; Segura Castruita, M.; González Fuentes, J. A y Valenzuela-García, J. R. 2016. Efecto del ácido salicílico en la producción y calidad nutracéutica de frutos de tomate. Rev. Mex. Cienc. Agric. 17(Pub. Esp.):3405-3414. 